Supplement of Saf. Nucl. Waste Disposal, 1, 263-264, 2021

https://doi.org/10.5194/sand-1-263-2021-supplement

(c) Author(s) 2021. CC BY 4.0 License.

Supplement of

\title{
Deep borehole disposal of intermediate-level waste
}

Dirk Mallants et al.

Correspondence to: Dirk Mallants (dirk.mallants@csiro.au)

The copyright of individual parts of the supplement might differ from the article licence. 


\section{Illi! cslio}

\section{Demonstrating Deep Borehole Disposal of Intermediate-Level Waste: Progress from Australia's RD\&D Project}

SafeND - 10-12 November, 2021

Dirk M allants and Team |

Australia's National Science Agency

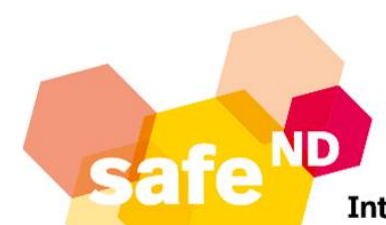

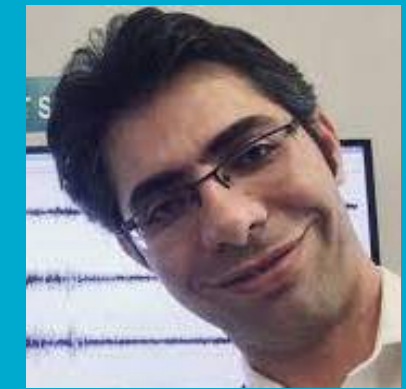
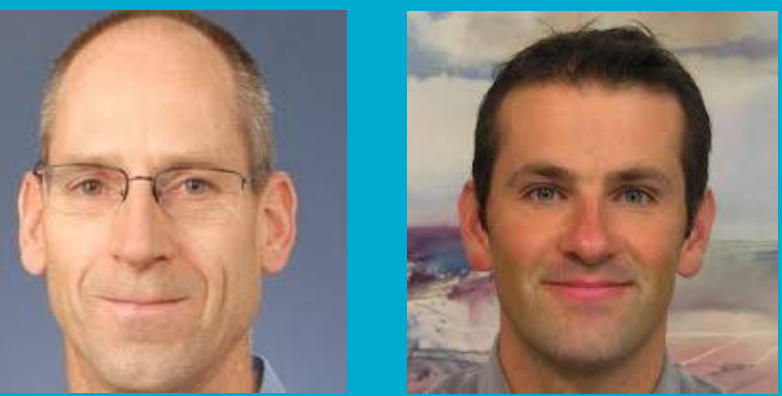

Yousef Beiraghdar Christian Doblin Lionel Esteban
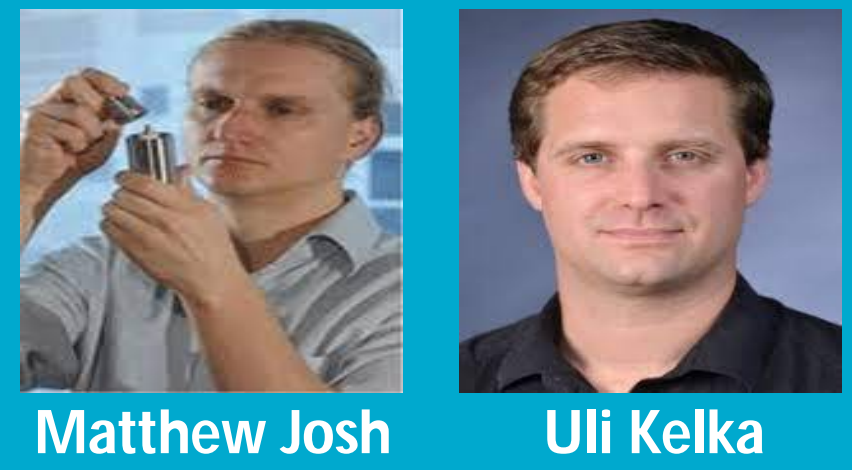

Uli Kelka

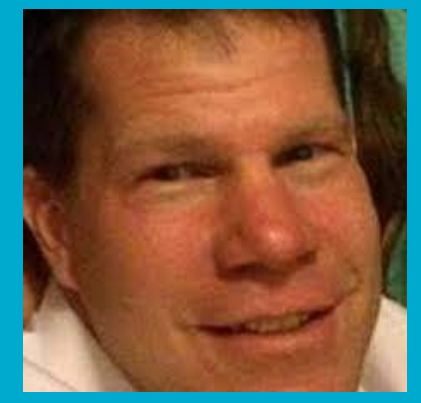

Peter Schaubs

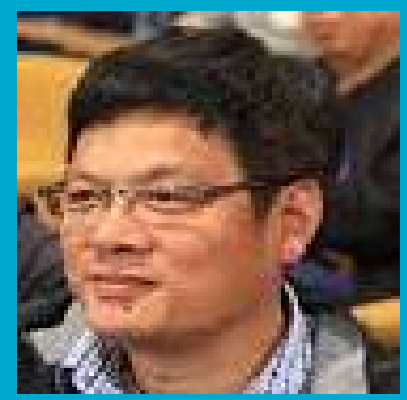

Baotang Shen
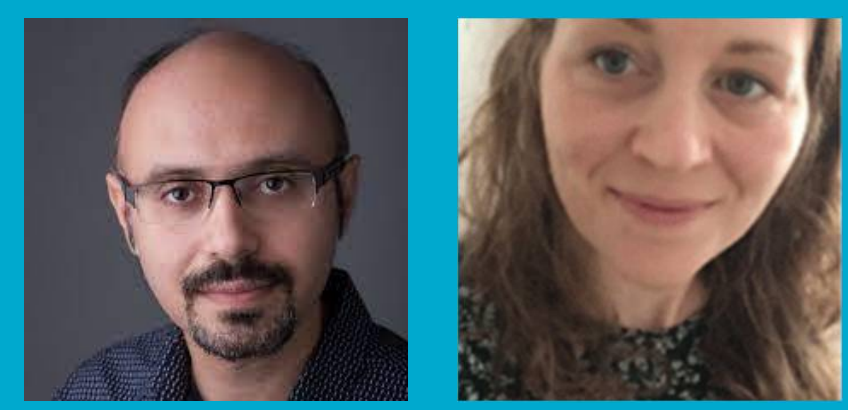

Kaveh L. Sookhak Cornelia Wilske
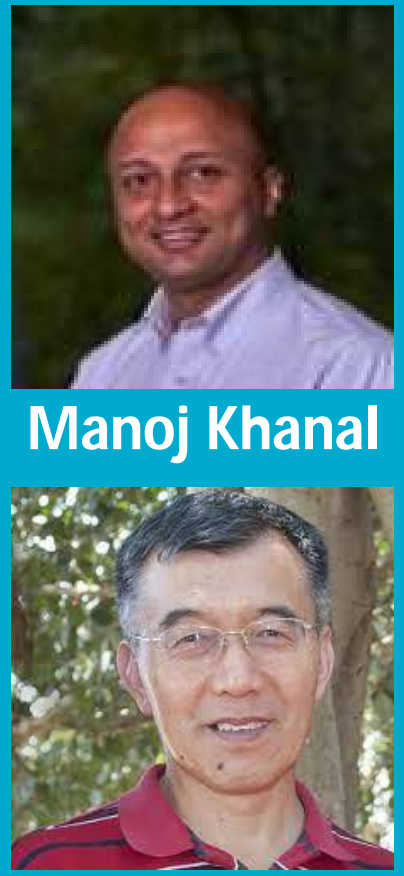

Jingyu Shi

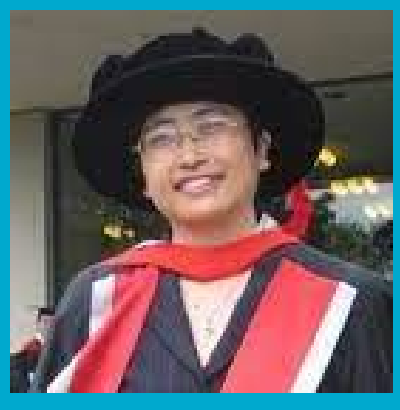

Junfang Zhang 
- Deep borehole disposal demonstration project

- Multi-barrier system for Deep Borehole Disposal

- Safety Functions

- Streamlining RD\&D

- Example RD\&D activities

$\checkmark$ Confirming diffusion dominated transport

$\checkmark$ Radionuclide sorption: M olecular Dynamics Simulation

$\checkmark$ Durable coatings for waste containers

$\checkmark$ Geological fault network analysis/FE mesh generator and fault modelling

$\checkmark$ Borehole mechanical stability modelling

$\checkmark$ Temperature evolution in borehole $\&$ host rock 
III) Deep borehole disposal csiRo demonstration project

Full-scale demonstration tests:

$\checkmark$ Characterisation borehole $(\sim 2000 \mathrm{~m})$

$\checkmark$ Drilling deep, large-diameter hole (0.7-m diameter, $-2000 \mathrm{~m}$ deep) $\checkmark$ Waste emplacement testing of dummy canister \& seal emplacement $\checkmark$ Proto-type waste package

Pre-/post-closure assessments:

$\checkmark$ Intermediate-level waste (glass, synroc) $\checkmark$ Various other waste types/forms $\checkmark$ Various scenarios $\checkmark$ Safety case
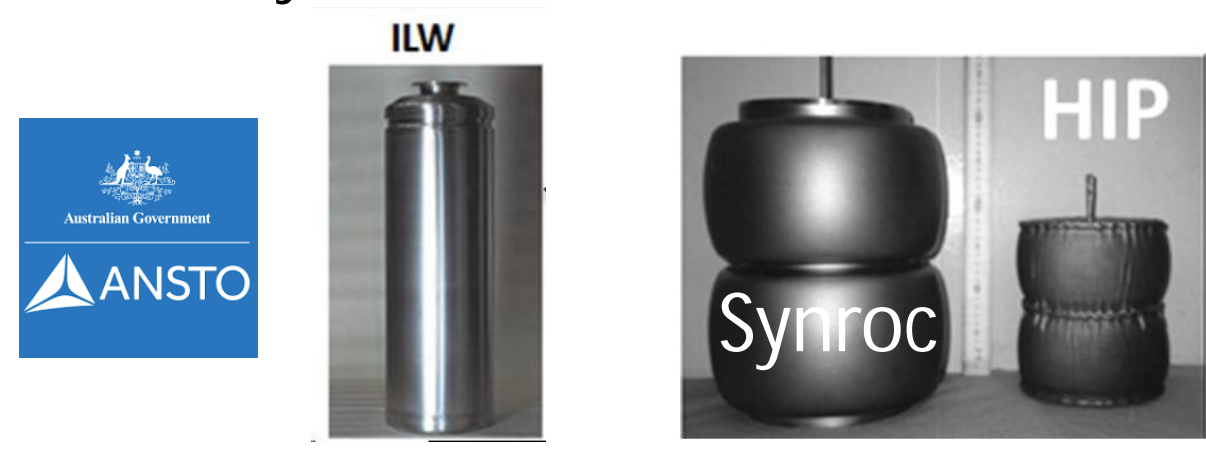

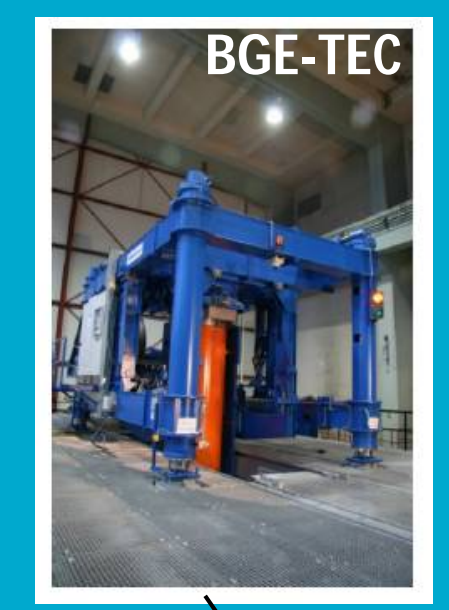

HAS-Innova rig
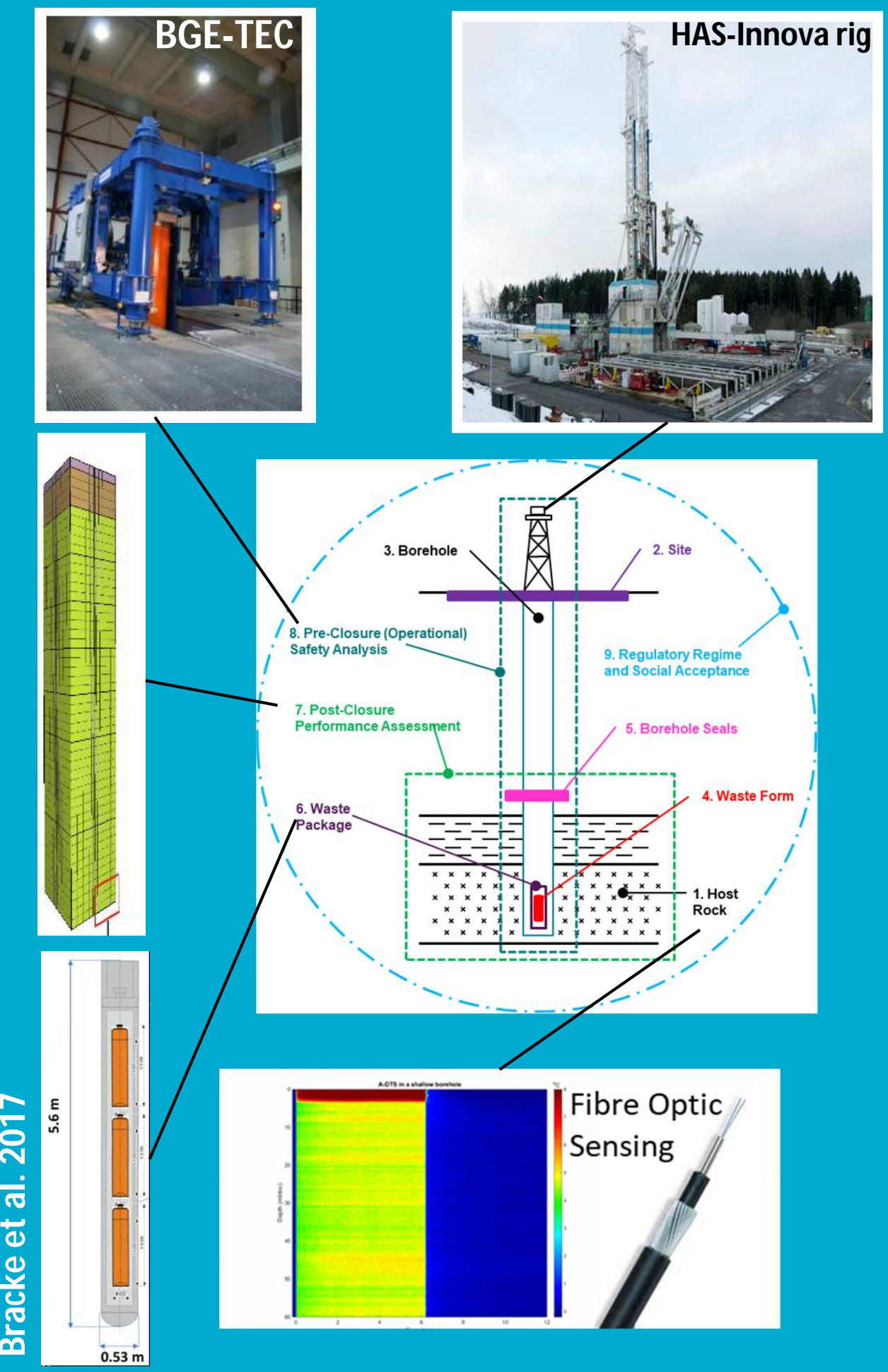
(IIII) Multiple-barrier system in a deep borehole Multiple Barrier System in a Deep Borehole Disposal Concept

\section{\#1 Glass matrix \\ $\square$ Isolation phase}

\#2 stainless steel primary package $\square$ Isolation phase
\#3 Disposal container

$\square$ Mild steel structural component

$\square$ Corrosion-resistant coating ( $\mathrm{Cu}, \mathrm{Ti}, . .$.

$\square$ Thermal phase
\#4 Borehole seals

$\square$ Cements

$\square$ Clays

$\square$ Crushed rock

$\square$ Isolation phase

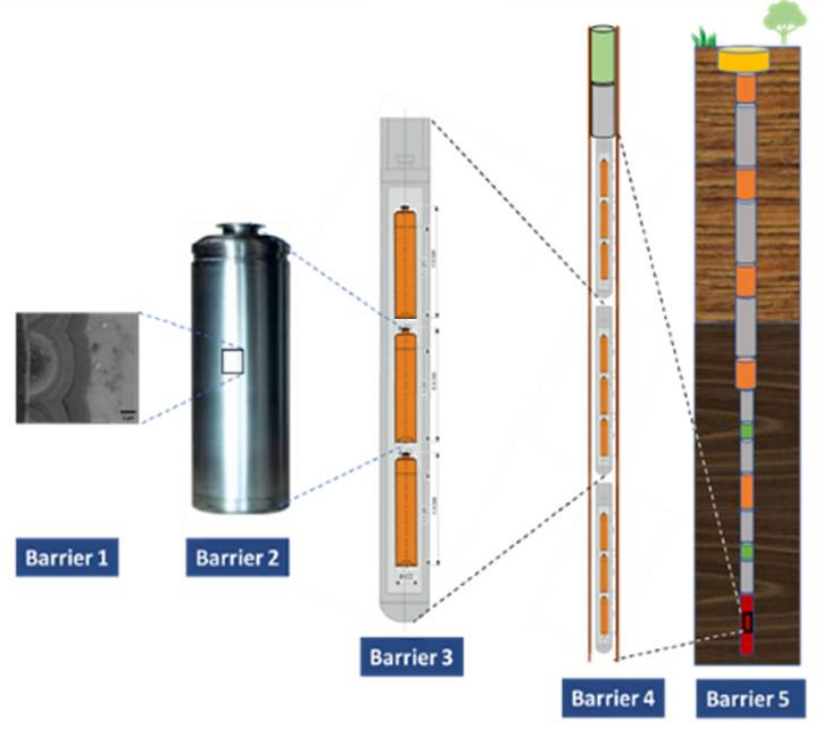

\#5 Geological environment

$\square$ Deep host rock

$\square$ Geological coverage

$\square$ Geological isolation phase 
(iIIII: M Multiple safety functions: I-C-R

\begin{tabular}{|c|c|c|c|c|c|}
\hline \multirow[b]{2}{*}{ Component } & \multirow[b]{2}{*}{$\begin{array}{l}\text { Isolation } \\
\text { (geology) }\end{array}$} & \multirow[b]{2}{*}{$\begin{array}{l}\text { Containment } \\
\text { (water } \\
\text { tightness) }\end{array}$} & \multicolumn{3}{|c|}{ Delay and attenuation of releases } \\
\hline & & & $\begin{array}{l}\text { Retardation-1 } \\
\text { (resistance to } \\
\text { leaching) }\end{array}$ & $\begin{array}{c}\text { Retardation-2 } \\
\text { (limiting water } \\
\text { ingress) }\end{array}$ & $\begin{array}{l}\text { Retardation-3 } \\
\text { (diffusion, } \\
\text { retention) }\end{array}$ \\
\hline Glass matrix & & & $\checkmark$ & & \\
\hline $\begin{array}{l}\text { SS primary } \\
\text { package }\end{array}$ & & $\checkmark$ & & $\checkmark$ & \\
\hline $\begin{array}{l}\text { Disposal } \\
\text { container }\end{array}$ & & $\checkmark$ & & $\checkmark$ & \\
\hline $\begin{array}{l}\text { Borehole } \\
\text { seals }\end{array}$ & $\checkmark \checkmark$ & & & $\checkmark \checkmark$ & $\checkmark \checkmark$ \\
\hline $\begin{array}{l}\text { Geological } \\
\text { environment }\end{array}$ & $\checkmark \checkmark \checkmark$ & & & $\checkmark \checkmark \checkmark$ & $\checkmark \checkmark \checkmark$ \\
\hline
\end{tabular}

$\checkmark \checkmark, \checkmark \checkmark \checkmark$ : scalable with depth

5 | Australia's National Science Agency 


\section{Iill: Streamlining RD\&D}

\section{Deep borehole disposal of ILW is}

technically feasible, safe and cost-effective

\begin{tabular}{|c|c|c|c|}
\hline & Safety Statements 目 & Feasibility Statements $\quad$ 目 & Confidence Enhancement \\
\hline 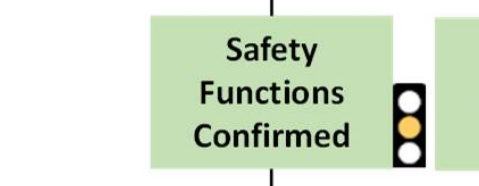 & 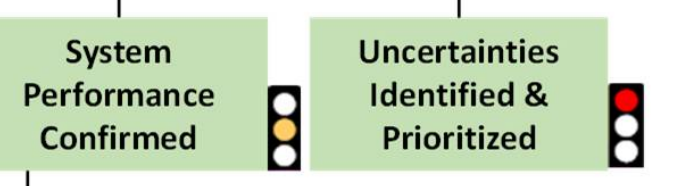 & 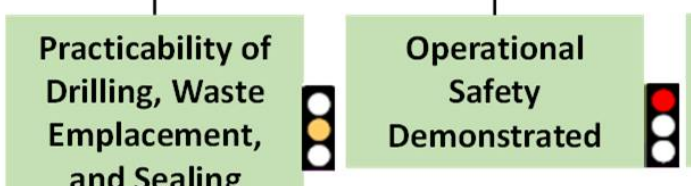 & 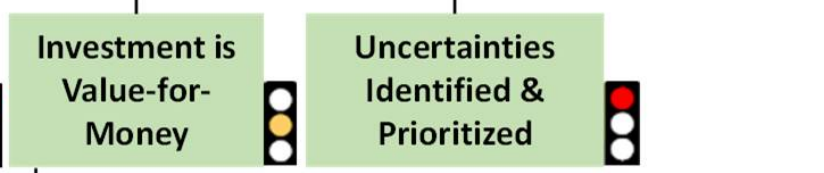 \\
\hline-8 & - smens & 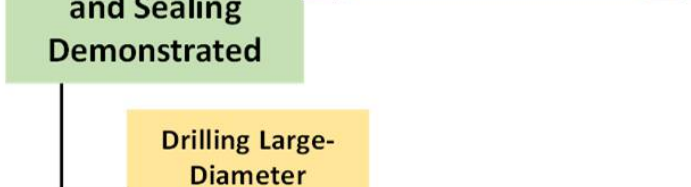 & F \\
\hline 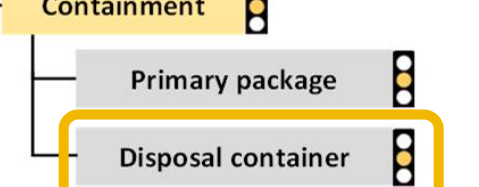 & E- & $=8$ & E \\
\hline mom 目 & 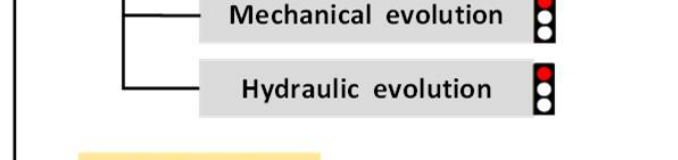 & 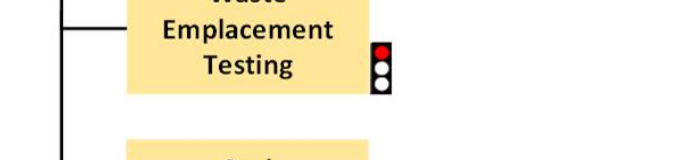 & 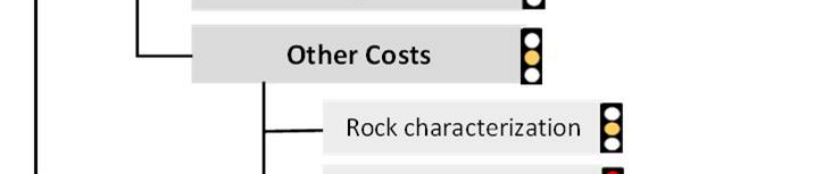 \\
\hline 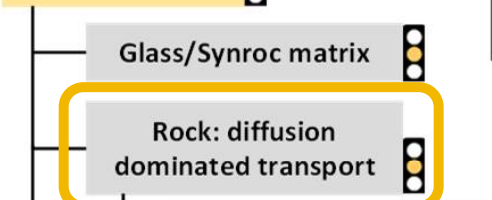 & and & - & max \\
\hline & $=0$ & 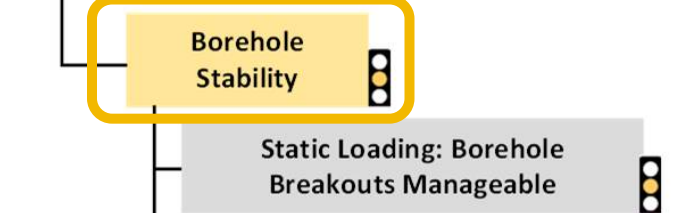 & 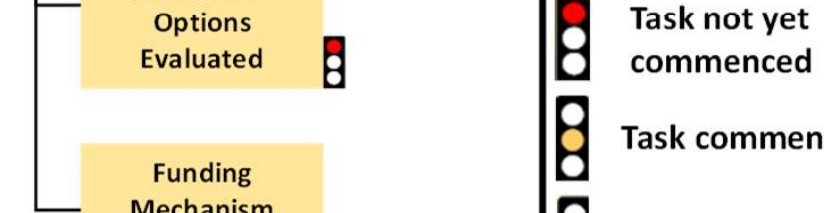 \\
\hline
\end{tabular}

Seals: diffusion

dominated transport

6 | Australia's National Science Agency 


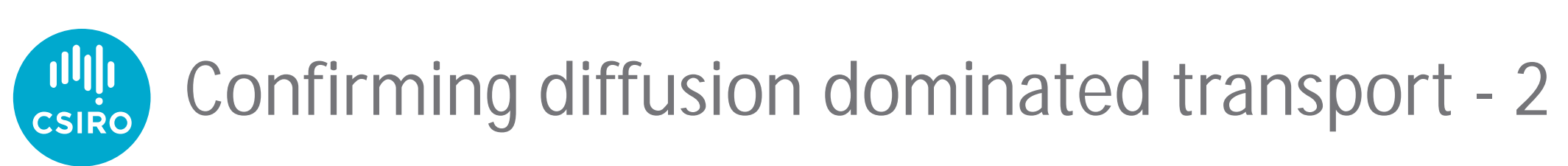

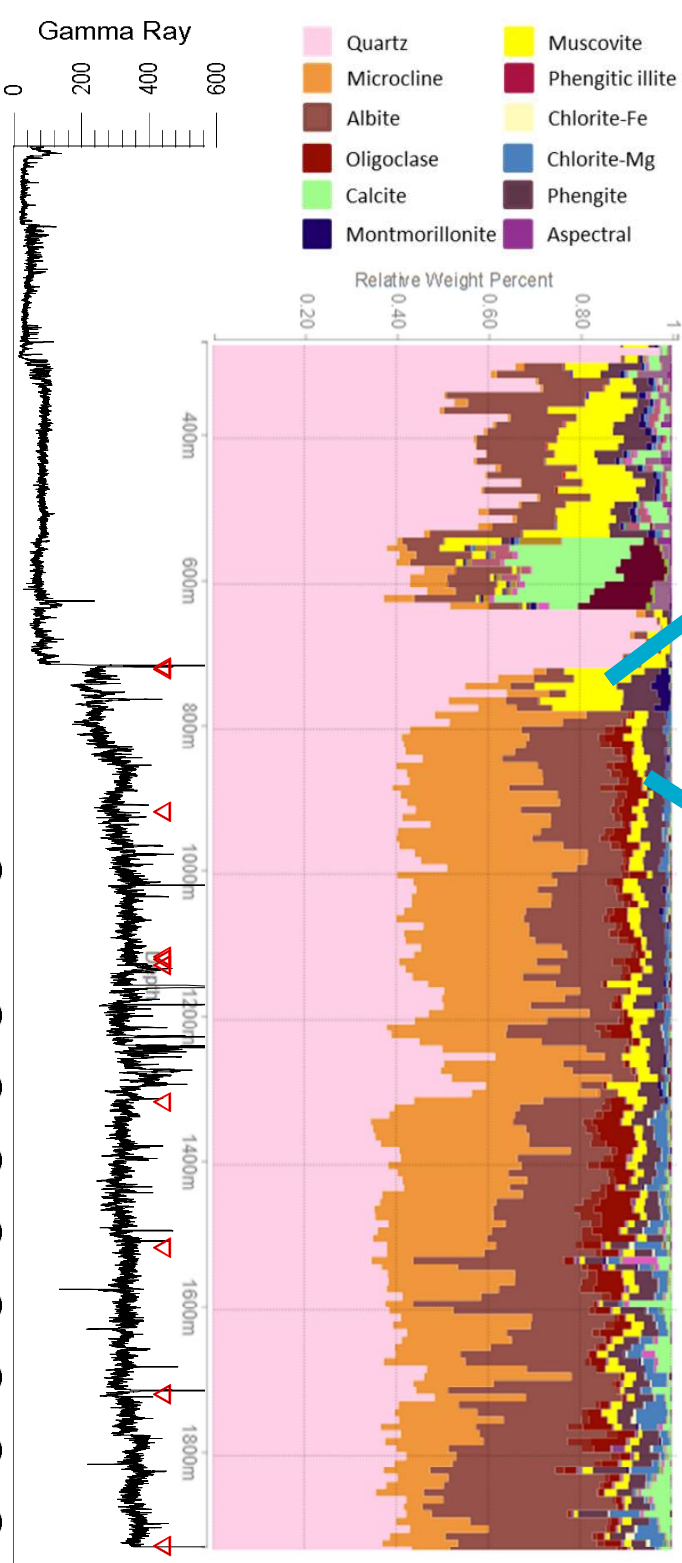

\section{Petrophysical properties}

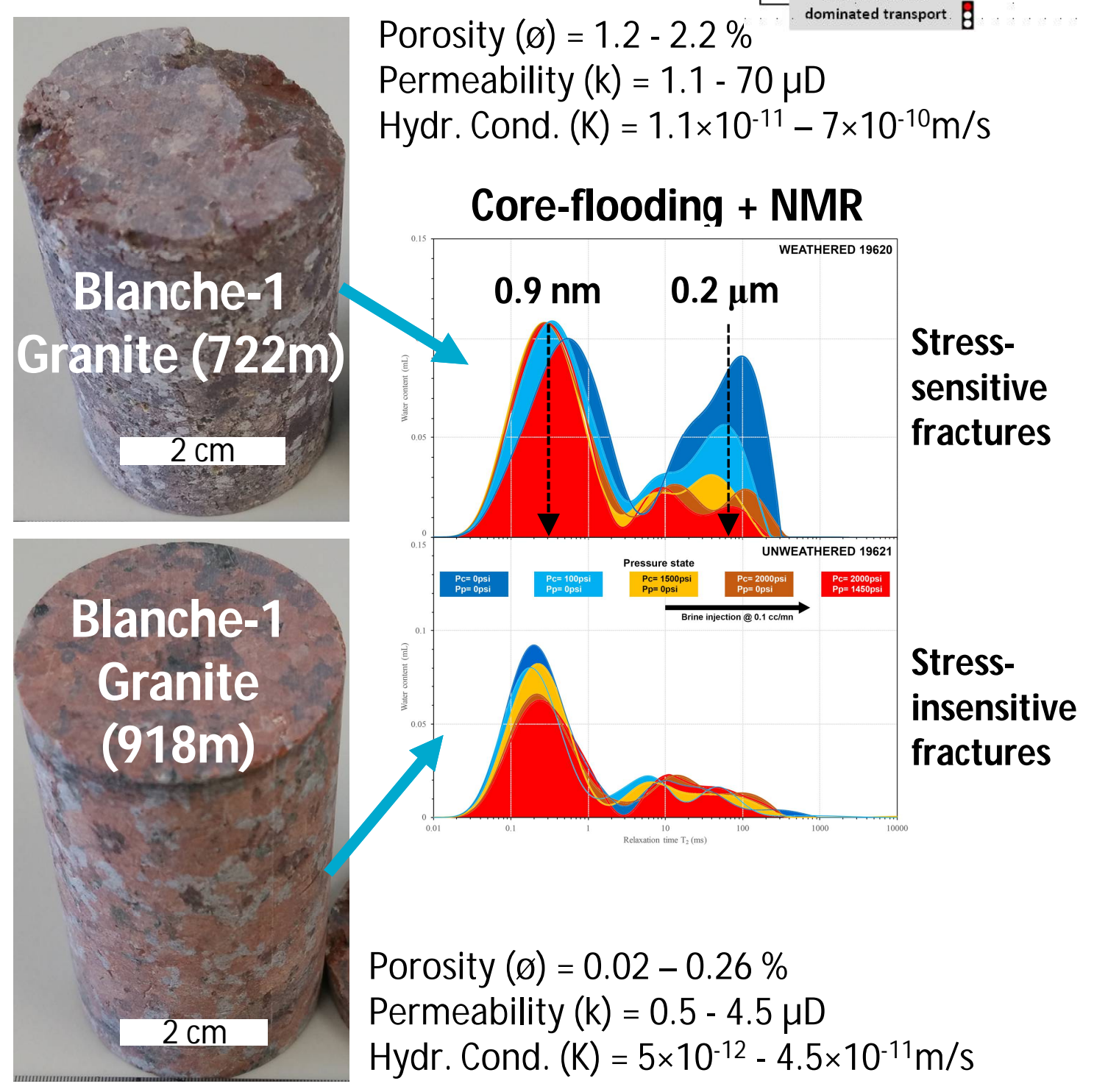

Hiltaba suite

granites

$\sim 1.575-1.6 \mathrm{Ga}$
Unweathered granite

Weathered granite

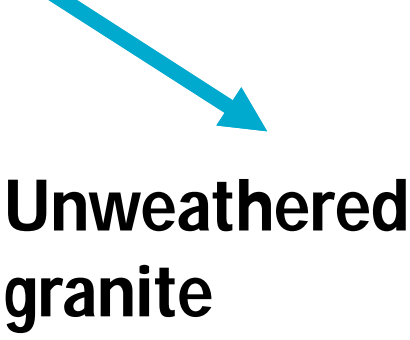




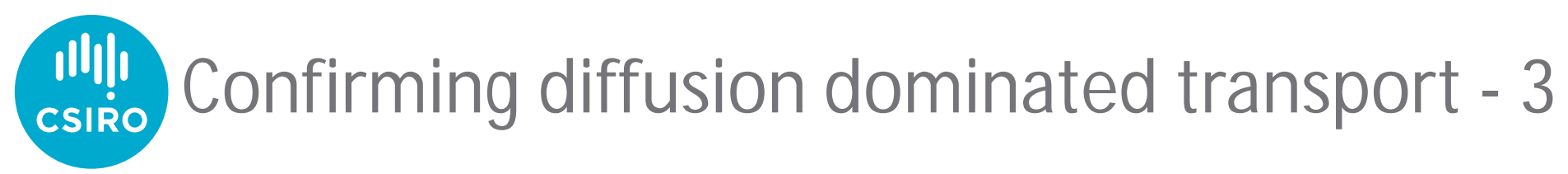

\section{- Environmental tracers (He, $\mathrm{Ne}, \mathrm{Ar}, \mathrm{Xe}$ )}

What are fluid inclusions?

- Trapped fluids inside minerals (voids in crystals)

- Heterogenous composition

- Gases, liquids and solids

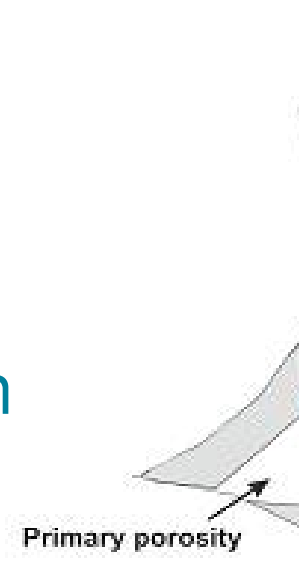

What can fluid inclusions tell us about host rock?

- How old are the fluids compared to the age of the rock? Ne \& Ar isotopes

- Any more recent processes involving fresh groundwater? $\mathrm{Ne} \& \mathrm{Ar}$ isotopes

- Provenance and residence time of groundwater (isolated system) grain
Primary inclusions

Aqueous and petroleum inclusions in quartz
overgrowth

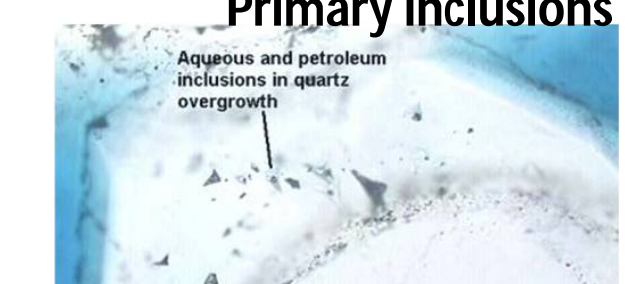

Detrital quart

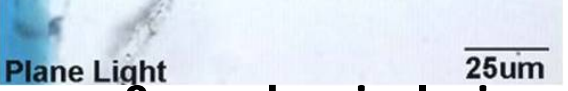

Secondary fluid inclusion

in micro - fracture trail

Secondary inclusions

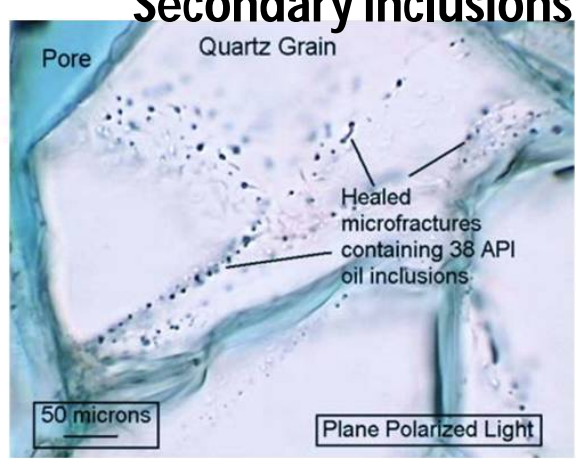

in quartz overgrowth cement

Melt inclusions

Plane Polarized Light

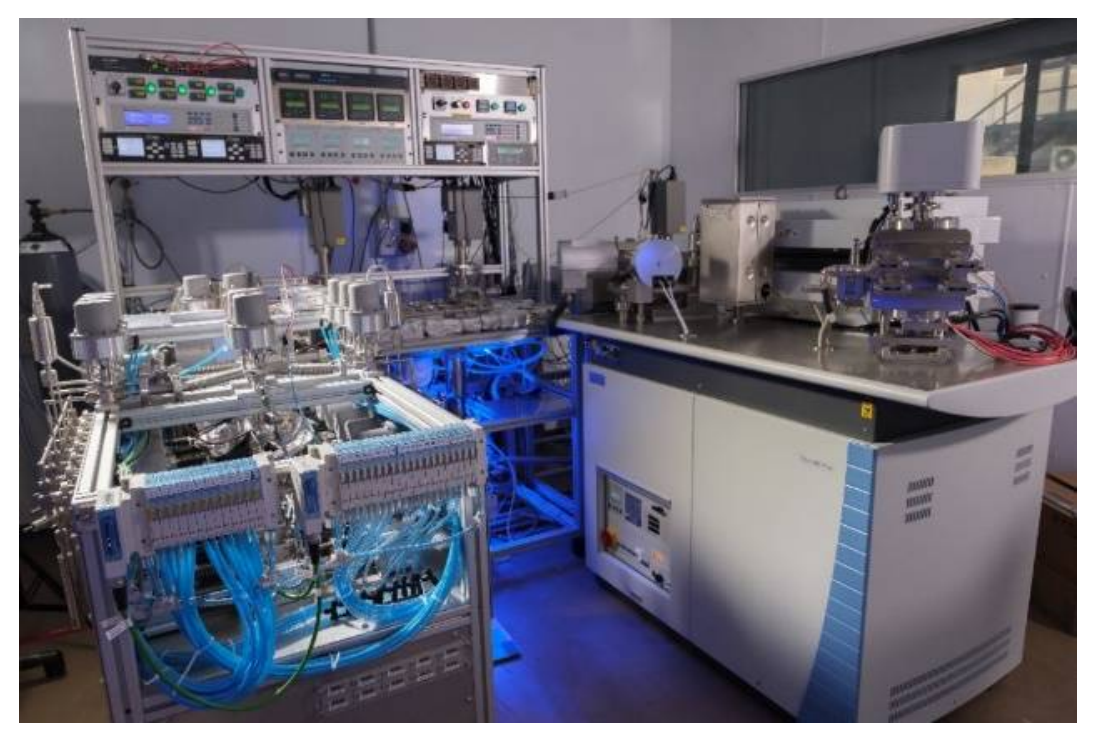

Fully automated, high throughput multiCollector noble gas mass spectrometer 

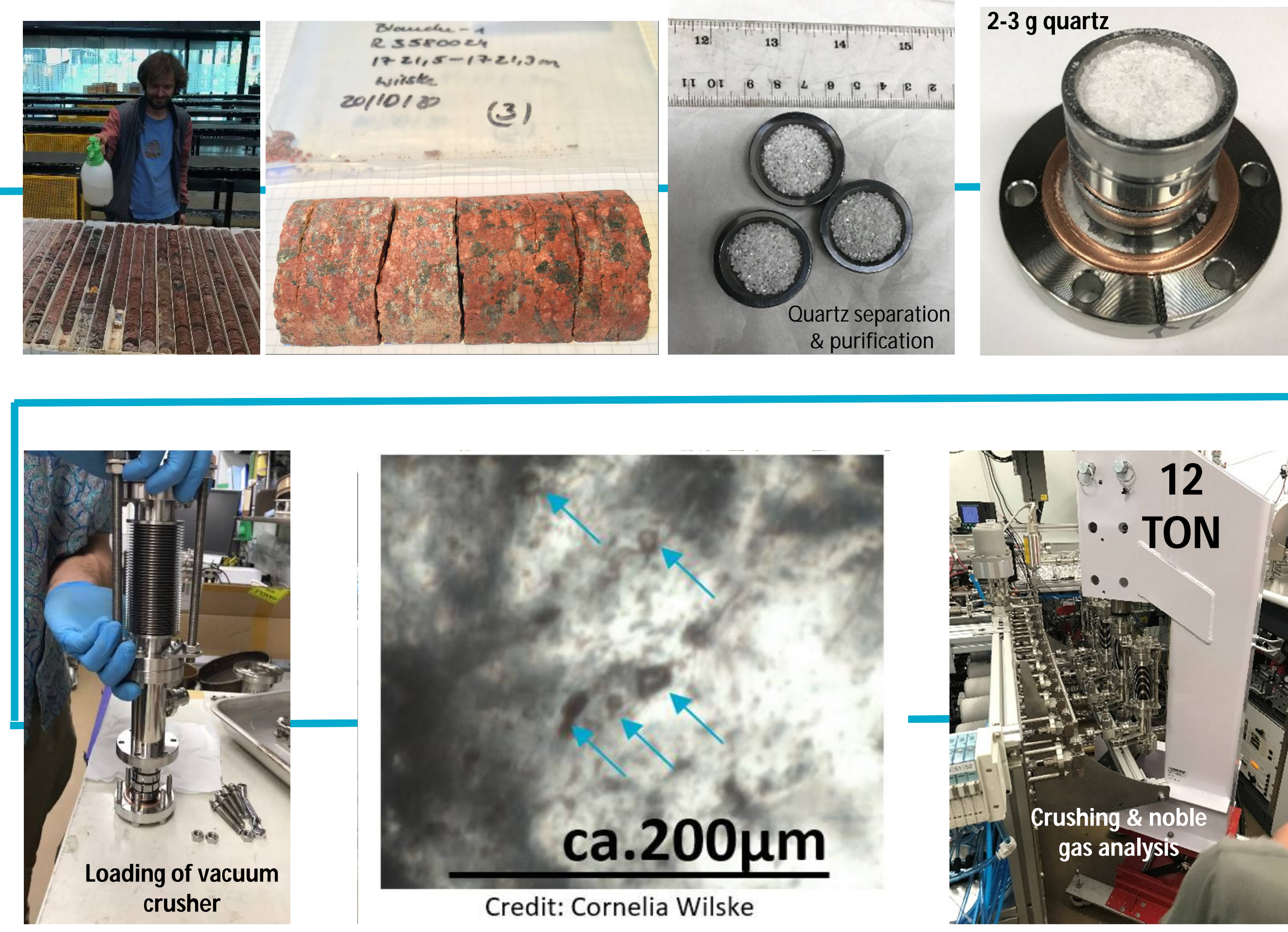

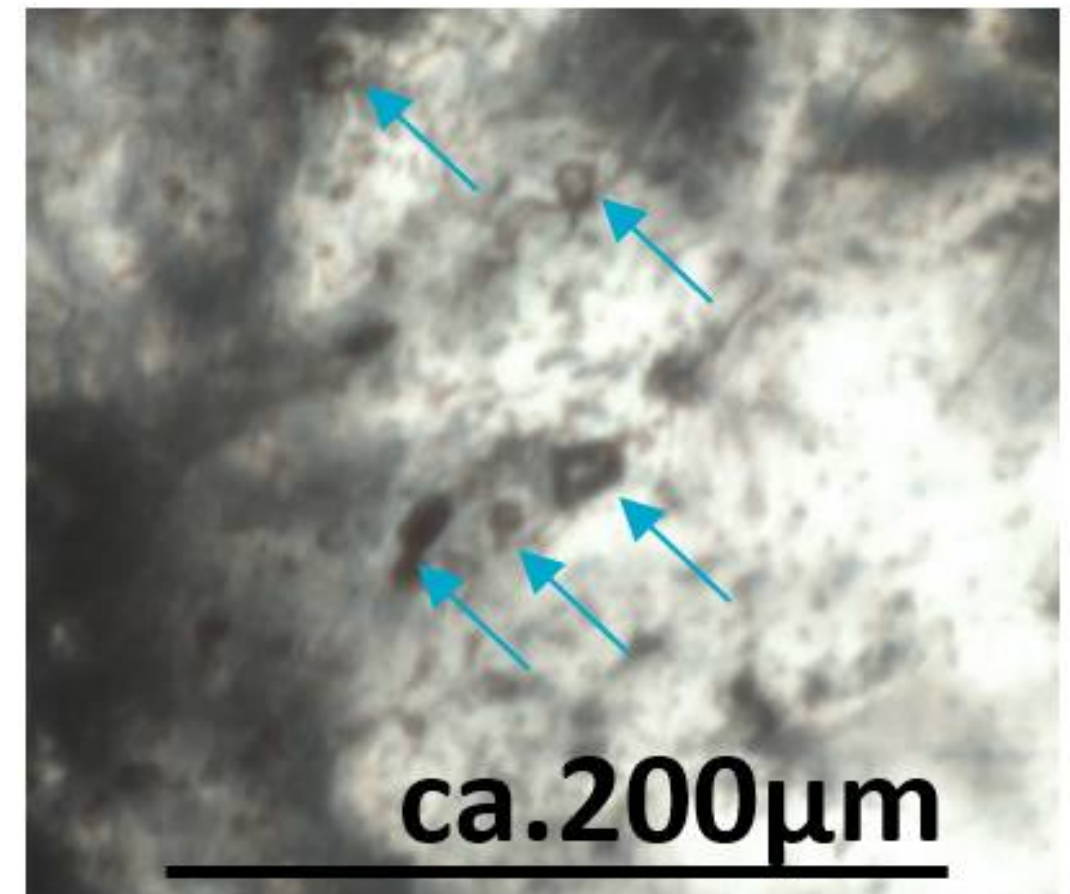

Credit: Cornelia Wilske

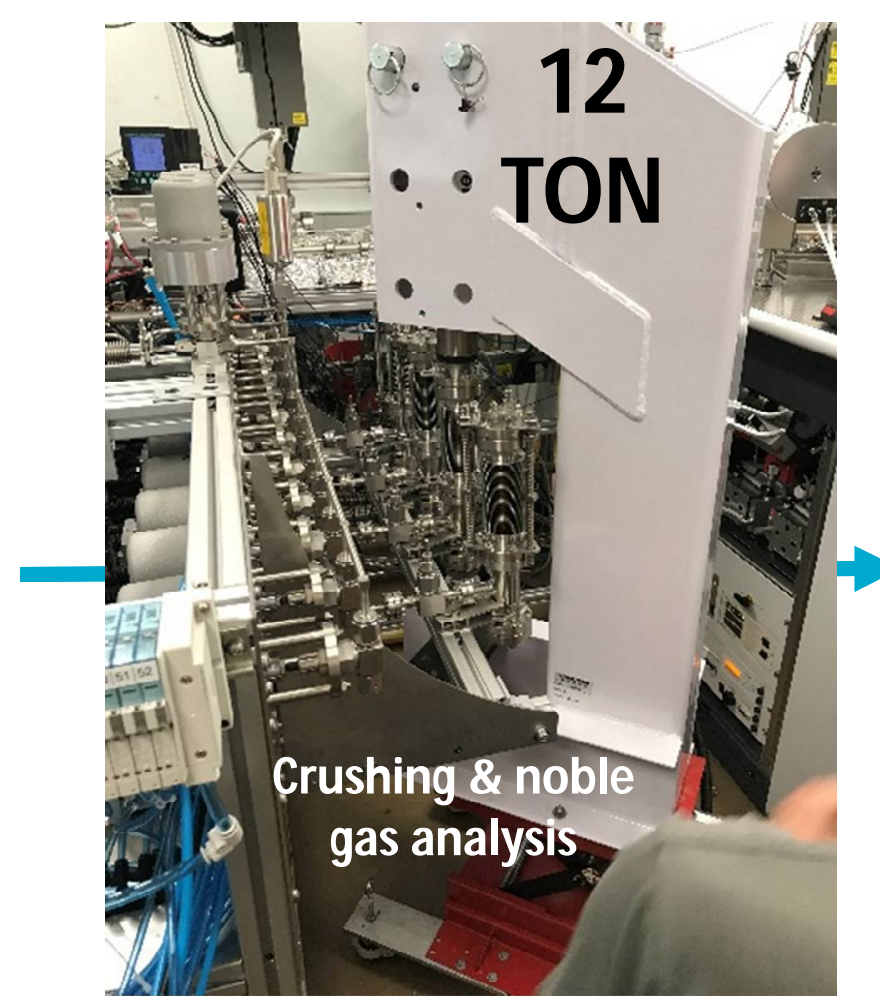




\section{IIII) Molecular Dynamics}

csiro Simulation of RN sorption

- Na-montmorillonite clay (Smectite)

- Aqueous solution: $\mathrm{Na}^{+}, \mathrm{CO}_{3}{ }^{2-}, \mathrm{UO}_{2}{ }^{2+}$

- 0.162 M uranyl carbonate

- $K_{D}$ calculated from atomic density profile

$$
K_{\mathrm{D}}=\left(\frac{C_{\text {adsorbed }}}{C_{\text {diffuse }}}\right)\left(\frac{V_{\mathrm{l}}}{m_{\mathrm{s}}}\right)
$$

- $\mathrm{K}_{\mathrm{D}}=58-151 \mathrm{~mL} / \mathrm{g}$ : similar to independent results from SANDIA (15 $200 \mathrm{~mL} / \mathrm{g}$ )

- Poly-nuclear U-species:

$\checkmark$ Adsorbed: $\left[\left(\mathrm{UO}_{2}\right)_{5}\left(\mathrm{CO}_{3}\right)_{5}\right]^{0}$

$\checkmark$ Aqueous: $\left[\mathrm{Na}_{2}\left(\mathrm{UO}_{2}\right)_{5}\left(\mathrm{CO}_{3}\right)_{6}\right]^{0}$
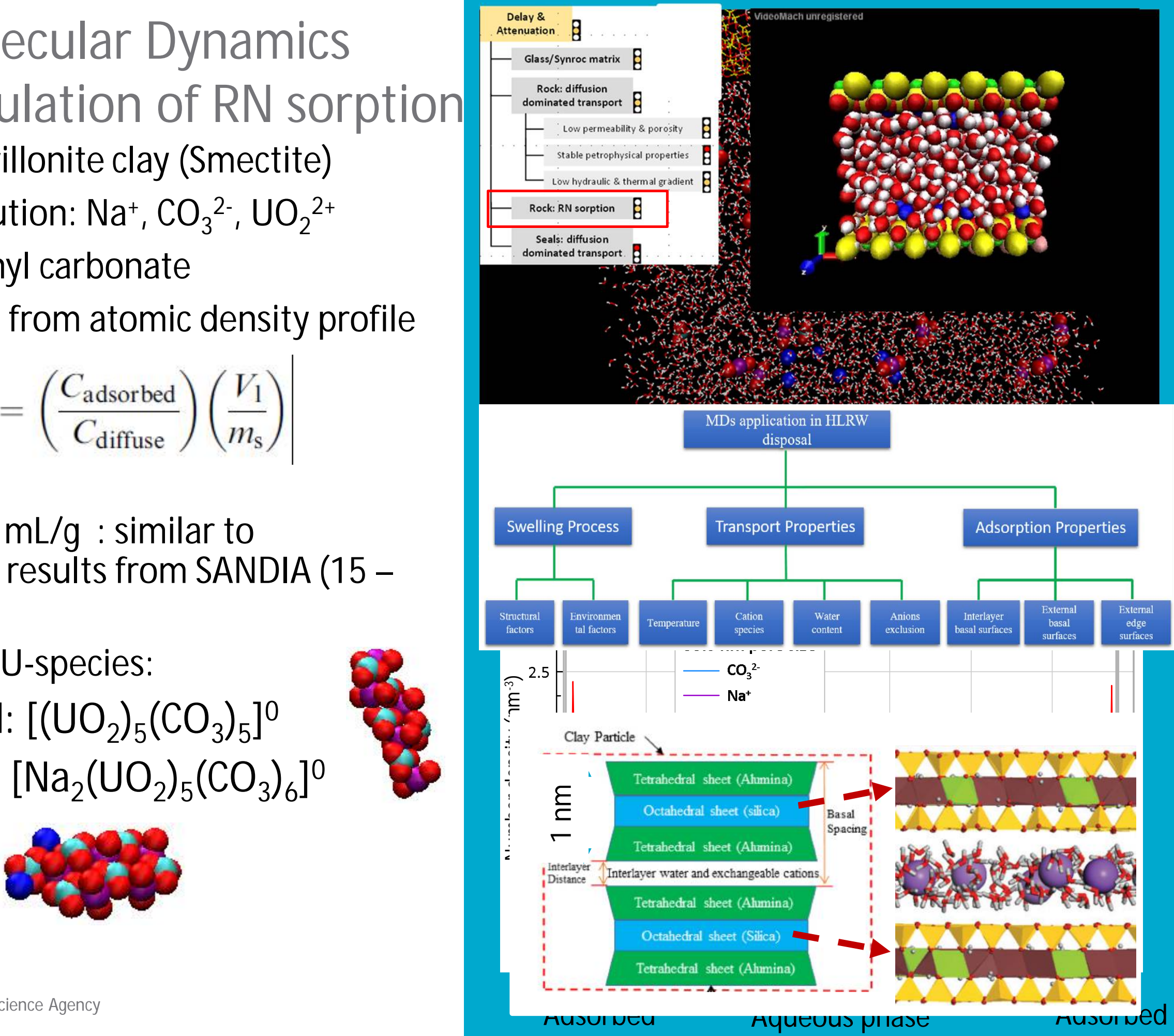
Iili!: Durable coatings for conatinment 目 (csiro disposal containers
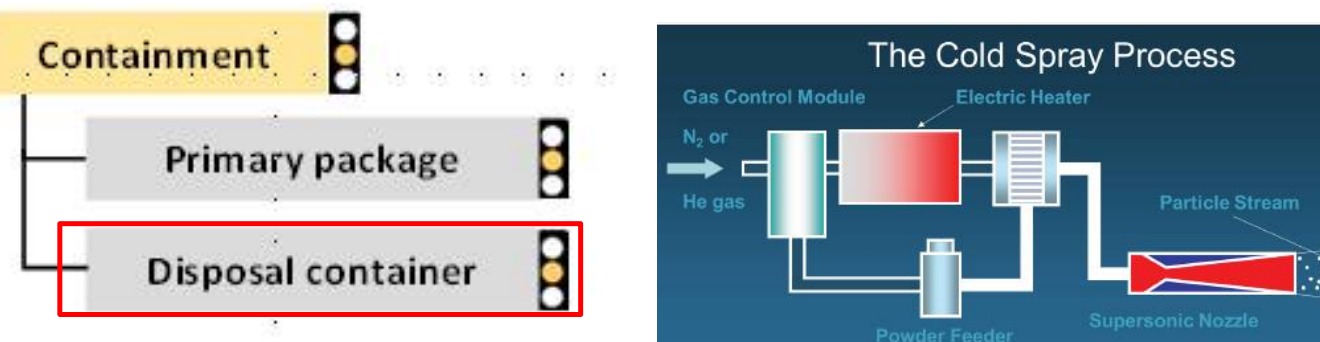

- Overpack/disposal container: mild structural steel + metal coating

- Literature review on coatings identified cold spray as most promising technology (minimal porosity, no high T effects)

- Cold spray uses supersonic particle velocities to deposit a dense layer of metallic powder on the surface of components

- Coating materials:

$\checkmark$ Titanium, Copper, Nickel, Chromium, Ni-Cr alloys, Tantalum

$\checkmark$ Composites (metal + ceramic)

- Benefits: surface property modification (corrosion and/or wear resistance)

- Corrosion \& Abrasion testing underway
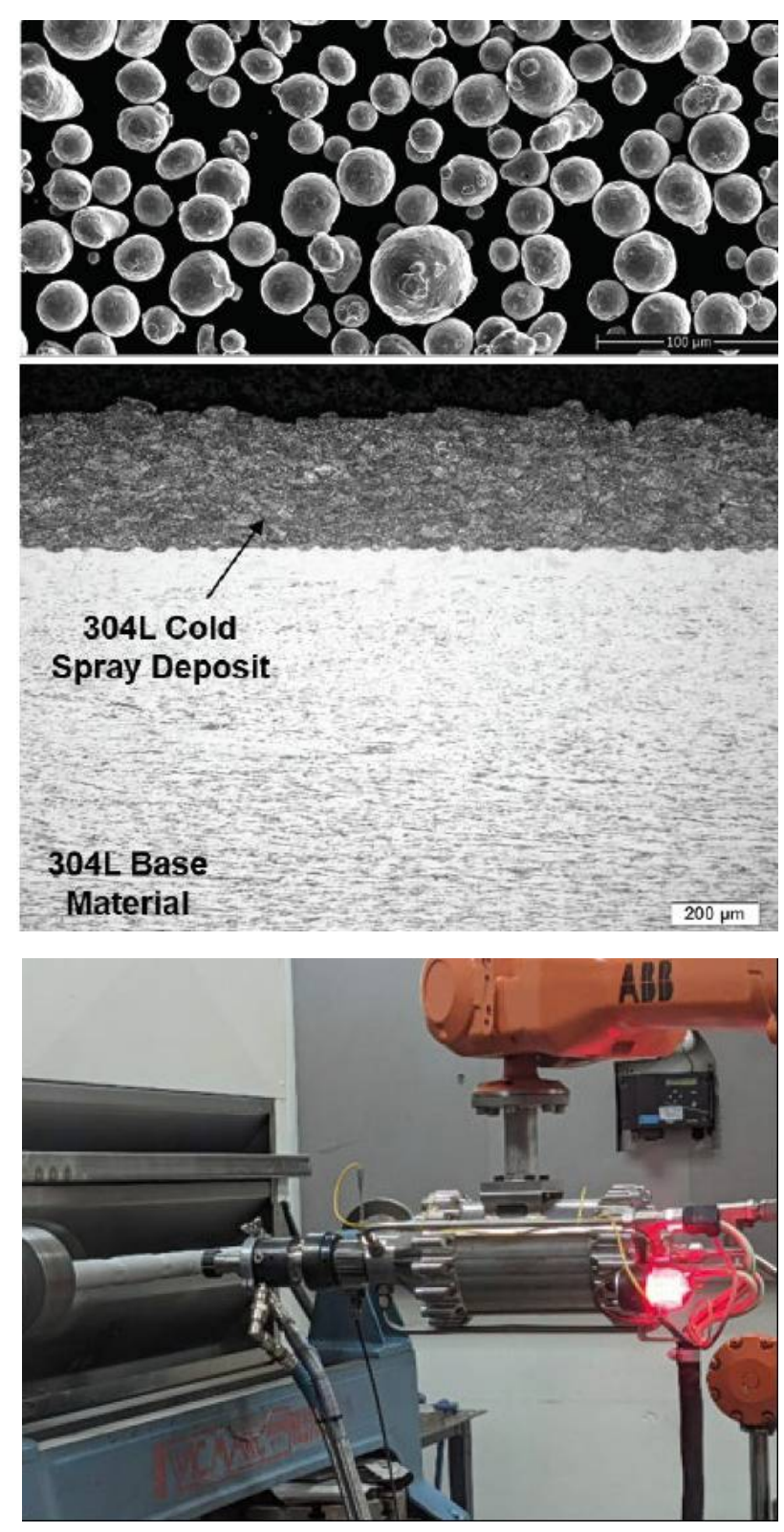
IIII!) Geological fault network analysis/ FE mesh generator

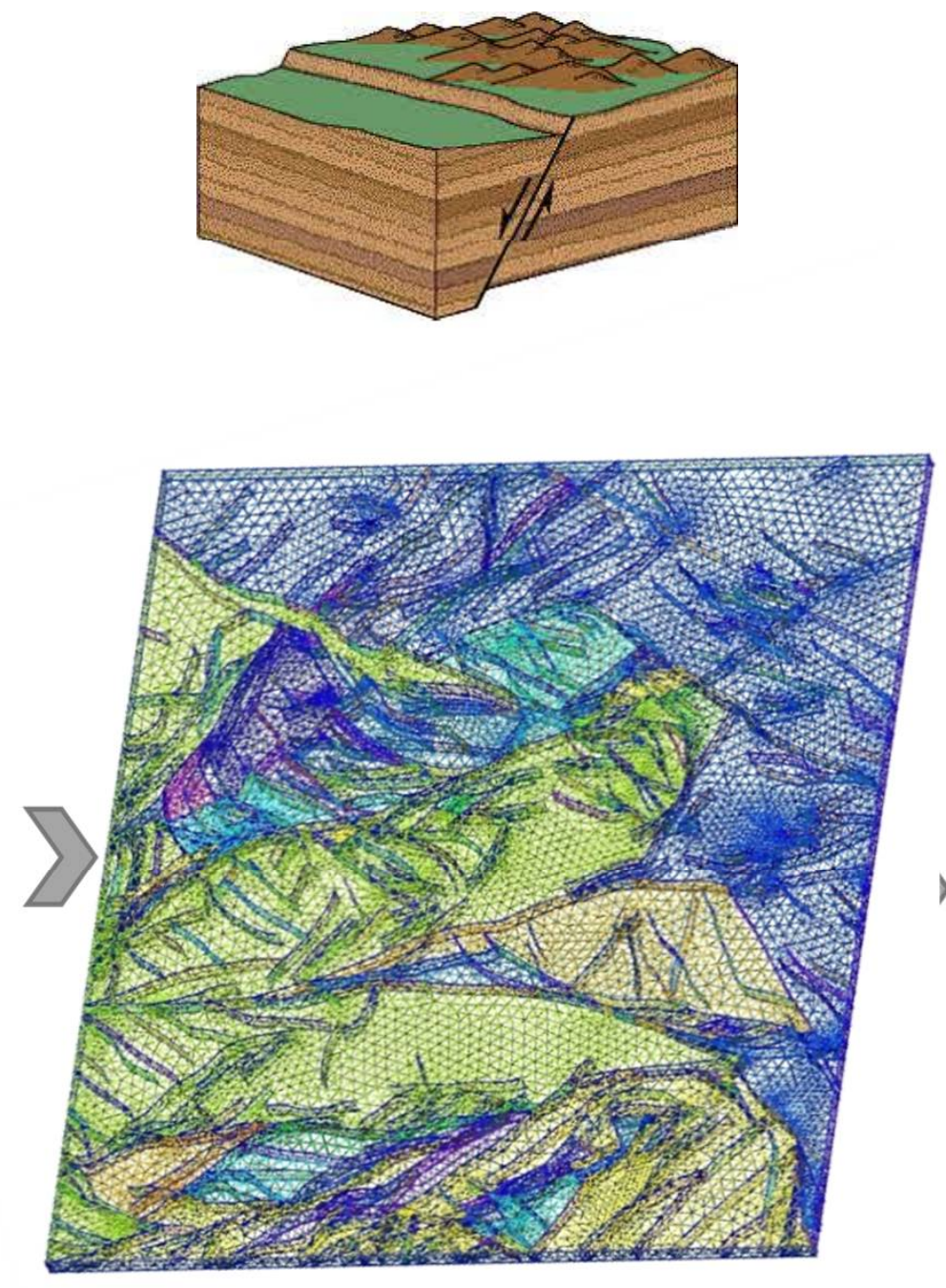

Automatic conversion in 2D finite element grid

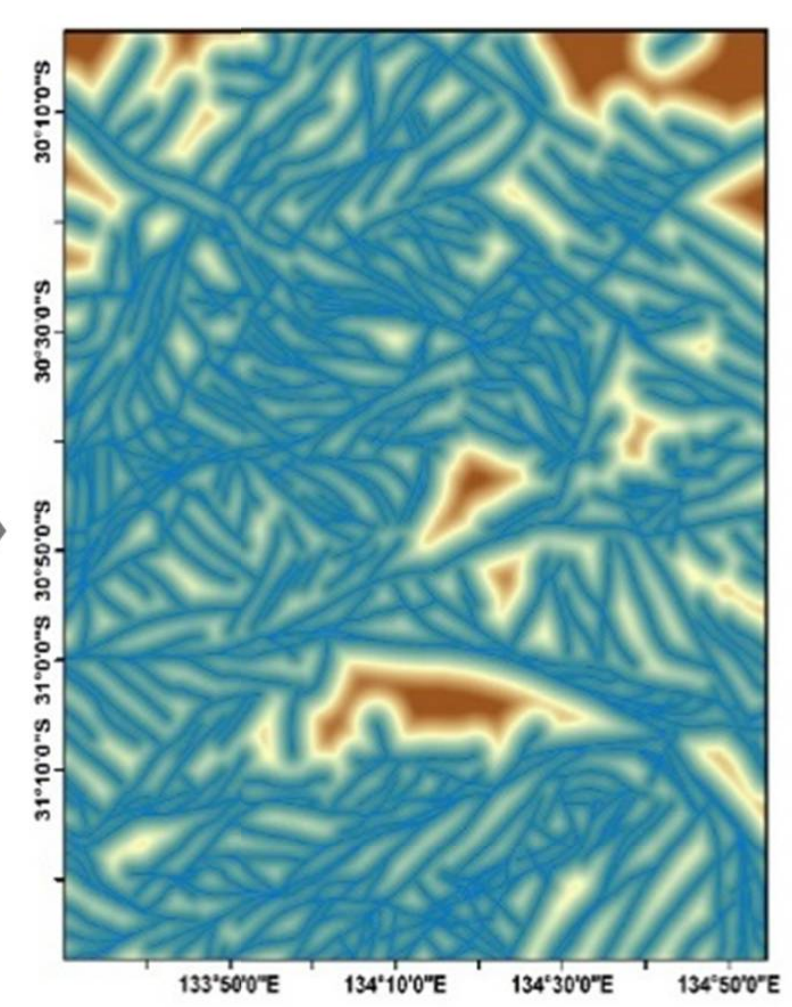

Calculated distance from faults + - 
(iil) Geological fault modelling: chemical transport

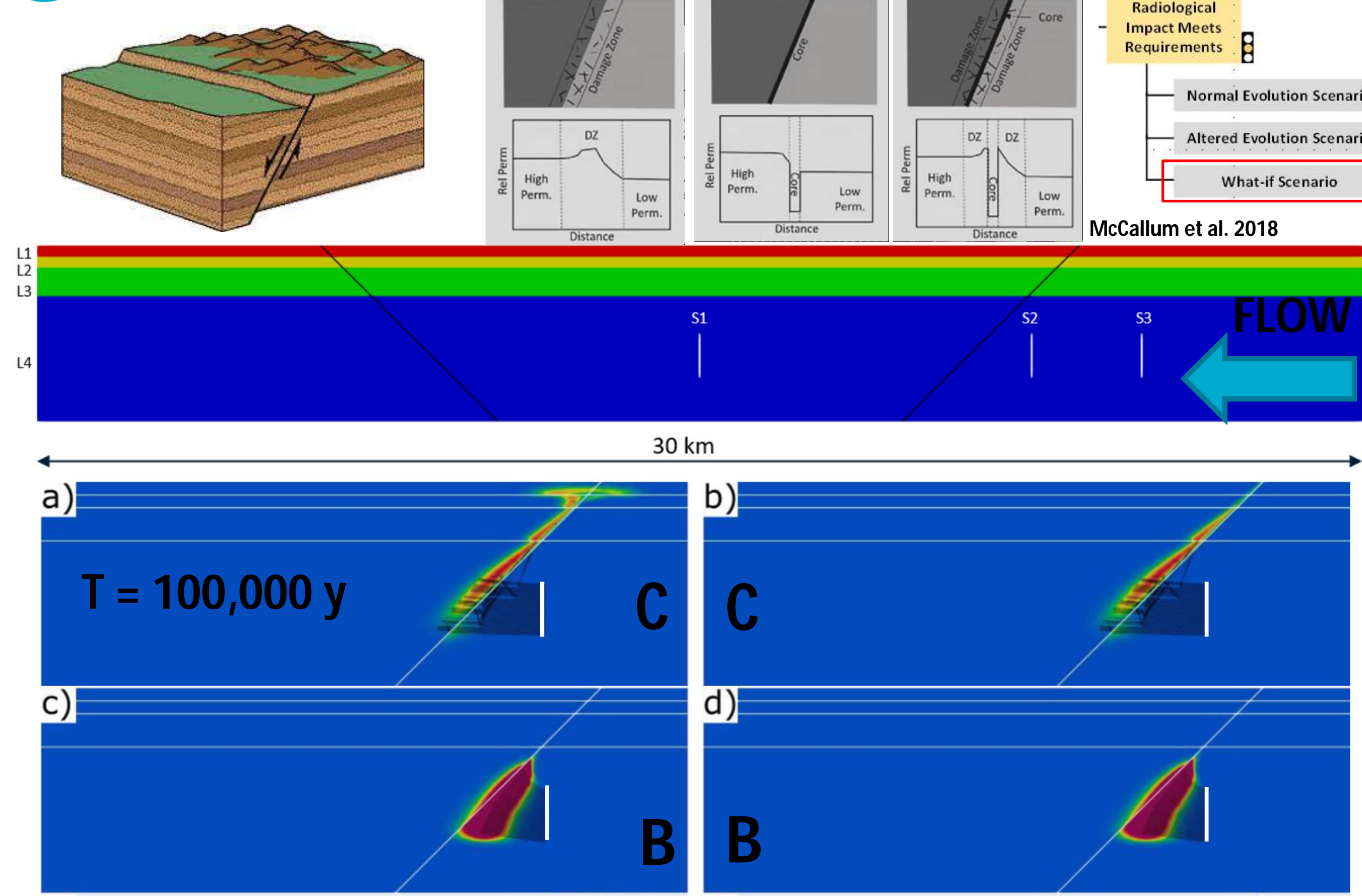




\section{(Iil) Temperature evolution in borehole $\&$ host rock}

- NF \& HR subject to perturbing processes:

\section{$\checkmark$ THMC}

- Thermal evolution from ILW

$\checkmark$ low heat load (50 W/canister)

$\checkmark$ sensitivity analysis

$>$ disposal depth

$>$ heat load

$>$ geothermal gradient

$>$ thermal properties

- TH evolution

$\checkmark$ Negligible convection

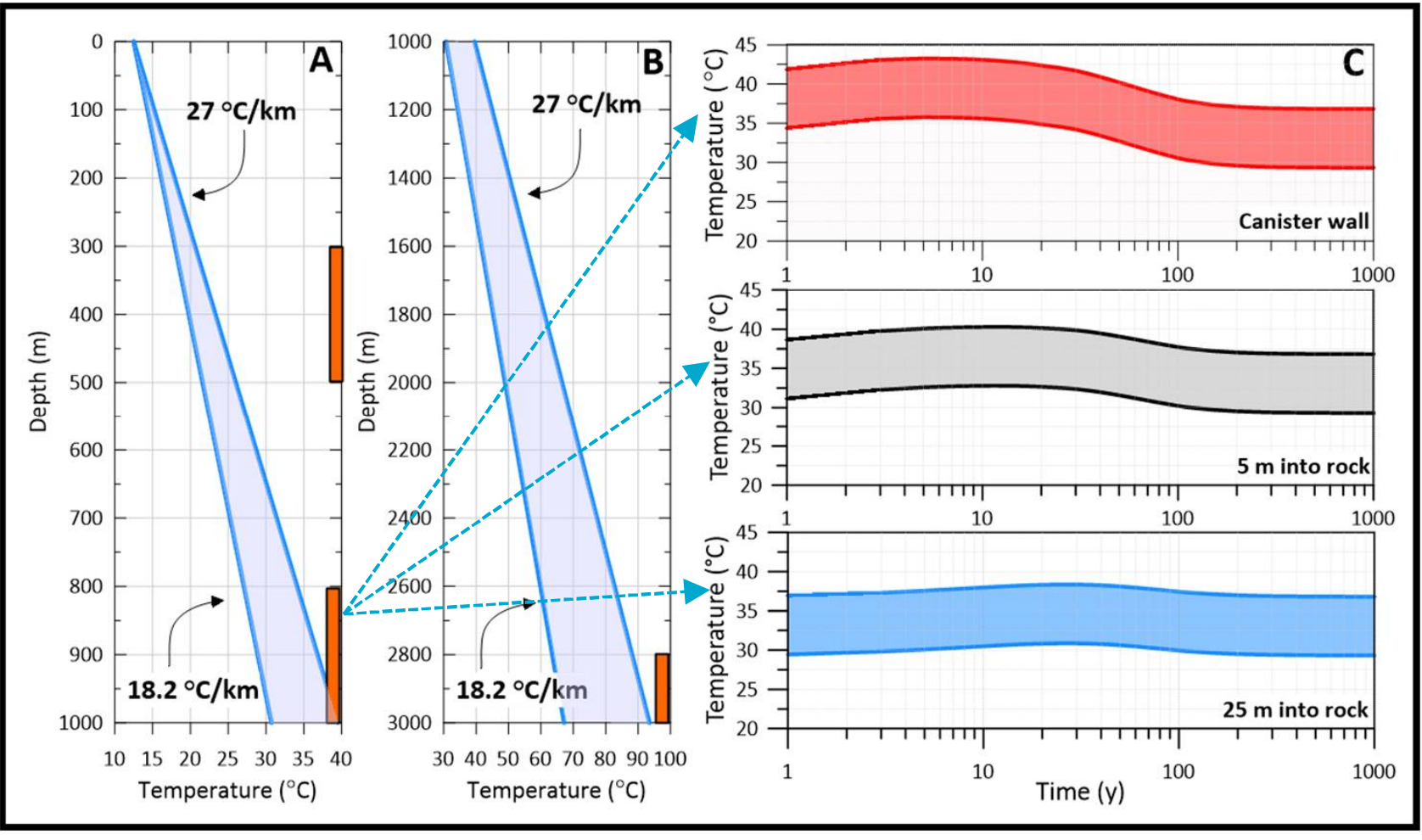


(illi) Borehole mechanical stability modelling

- Borehole breakout simulations using FRACOD

- Field-data available

- Exploratory analyses

$\checkmark$ Effect of rock strength

$\checkmark$ Effect of heating/ cooling

$\checkmark$ Effect of pre-existing fracture networks + effect on permeability

a)

Breakouts at $2000 \mathrm{~m}$ depth - granite

b)

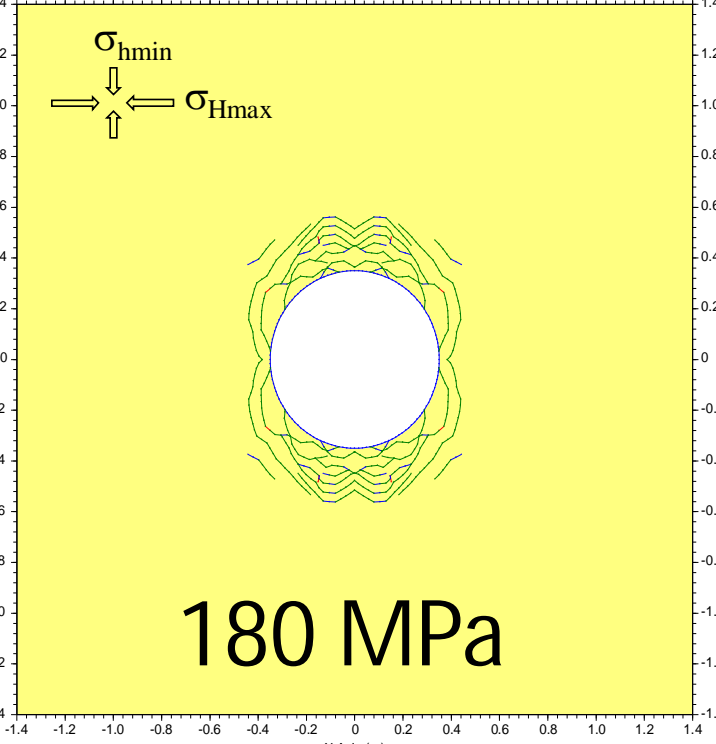

c)

Effect of cooling

\section{$120 \mathrm{MPa}$}

d)

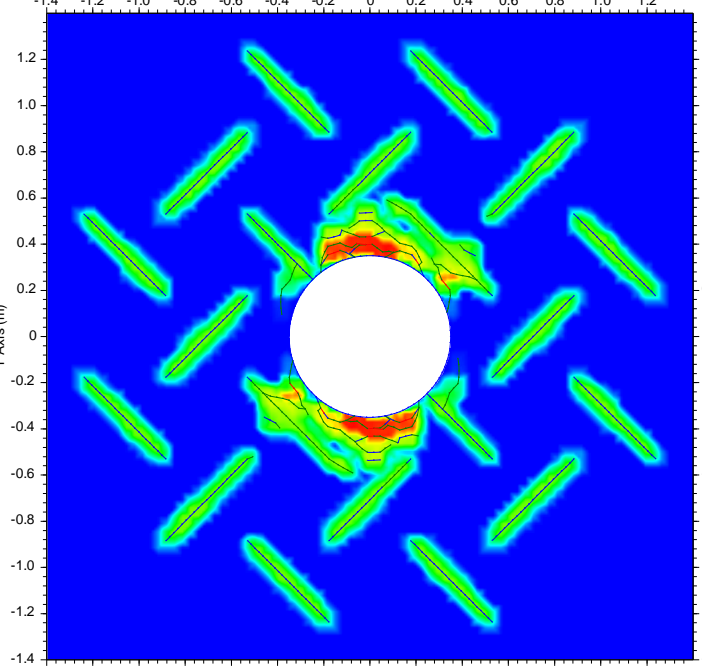


Ill

caiRo

Our Noble Gases on Record [explanatory video of CSIRO's noble gas capability in the groundwater sciences]

Thank you

Land and Water

Dr. Dirk Mallants

+61 883038595 dirk.mallants@csiro.au csiro.au/landandwater

Australia's National Science Agency 


\section{References}

1. Doblin, C., 2021. Review of corrosion resistant coating technologies for nuclear waste disposal canisters. Technical Report, CSIRO, Canberra, Australia.

2. Mallants D., Y. Beiraghdar, 2021a. Heat transport in the near field of a deep vertical disposal borehole: preliminary performance assessments. In: Waste Management Symposium, March 7-11, Phoenix, Arizona, USA.

3. M allants D., Y. Beiraghdar, 2021b. Radionuclide transport and deep borehole disposal: preliminary safety assessments. In: Waste M anagement 2021 Symposium; 7-11 March 2021; Phoenix, Arizona, USA.

4. Mallants D., Sander R., Avijegon A., Engelhardt, H.-J., 2021c. Cost analysis of deep large-diameter drill holes. In: Waste Management 2021 Symposium; 7-11 March 2021; Phoenix, Arizona, USA.

5. Schaubs, P., Kelka, U., 2021. Fault conceptualisation and numerical simulation of safe waste disposal sites. Technical Report, CSIRO, Canberra, Australia.

6. Shen, B., Shi, J., Khanal, M., M allants, D., 2022. Geomechanical M odelling of Borehole Stability for Deep Borehole Radioactive Waste Disposal, Waste Management Symposium2022, Phoenix, Arizona 2022 (submitted).

7. Sookhak Lari, K., M allants, D., 2021. A Darcy-scale coupled heat-mass transport model to assess radionuclide migration from deep disposal boreholes. J. Hazardous M aterials (in review).

8. Wilske, C., Suckow, A.O., Deslandes, A., Crane, P., Gerber, C., Spooner, N., Mallants, D., 2021. Fluid inclusions in minerals of the deep crust-Investigations with the new high vacuum crushing system at the CSIRO noble gas facility, Proc. Goldschmidt2021 Conference, virtual.

9. Zhang. J., M allants, D., Brady, P.V., 2021. M olecular Dynamics Study of Uranyl Adsorption from Aqueous Solution to Smectite, Appl. Clay Sci. 2021 (in review). 